\title{
Construction of the miRNA-mRNA regulatory network and analysis of hub genes in oral squamous cell carcinoma
}

\author{
Zifeng Cui ${ }^{\mathrm{a}}$, Qiwen Song ${ }^{\mathrm{b}}$, Yanping Chen ${ }^{\mathrm{a}}$, Kaicheng Yang ${ }^{\mathrm{a}}$
}

\begin{abstract}
Background. Oral squamous cell carcinoma (OSCC) severely affects the quality of life and the 5-year survival rate is low. Exploring the potential miRNA-mRNA regulatory network and analyzing hub genes and clinical data can provide a theoretical basis for further elucidating the pathogenesis of OSCC.

Methods. The miRNA expression datasets of GSE1 13956 and GSE124566 and mRNA expression datasets of GSE31056, GSE37991 and GSE13601 were obtained from the Gene Expression Omnibus databases. The differentially expressed miRNAs (DEMs) and mRNAs (DEGs) were screened using GEO2R. Gene ontology (GO) and Kyoto Encyclopedia of Genes and Genomes (KEGG) enrichment analyses were performed by DAVID database. The PPI network was established through STRING database and the hub genes were preliminarily screened out by Cytoscape software. After identifying the hub genes in the TCGA database, we predicted the potential DEM transcription factors, constructed a miRNA-mRNA regulatory network, and analyzed the relationship between the hub genes and clinical data.

Results. A total of 28 DEMs and 764 DEGs were screened out, which were composed of 285 up-regulated genes and 479 down-regulated genes. Enrichment analysis showed that up-regulation of DEGs were mainly enriched in extracellular matrix organization and cancer-related pathway, while down-regulation of DEGs were mainly enriched in muscular system process and adrenaline signal transduction. After preliminary screening by PPI network and identification in TCGA, the up-regulated FN1, COL1A1, COL1A2, AURKA, CCNB1, CCNA2, SPP1, CDC6, and down-regulated ACTN2, TTN, IGF1, CAV3, MYL2, DMD, LDB3, CSRP3, ACTA1, PPARG were identified as hub genes. The miRNA-mRNA regulation network showed that hsa-miR-513b was the DEM with the most regulation, and COL1A1 was the DEG with the most regulation. In addition, CDC6, AURKA, CCNB1 and CCNA2 were related to overall survival and tumor differentiation.

Conclusions. The regulatory relationship of hsa-miR-513b/ CDC6, CCNB1, CCNA2 and the regulatory relationship of hsa-miR-342-5p /AURKA were not only verified in the miRNA-mRNA regulatory network but also related to overall survival and tumor differentiation. These results indicated that they participated in the cellular regulatory process, and provided a molecular mechanism model for the study of pathogenesis.
\end{abstract}

Key words: bioinformatics, oral squamous cell carcinoma (OSCC), regulatory network

Received: June 5, 2021; Revised: December 23, 2021; Accepted: January 19, 2022; Available online: Fabruary 3, 2022

https://doi.org/10.5507/bp.2022.001

(c) 2022 The Authors; https://creativecommons.org/licenses/by/4.0/

${ }^{a}$ Department of Stomatology, The Fourth Hospital of Hebei Medical University, No. 12, Jiankang Road, Shijiazhuang 050000, Hebei, China 'Oral and Maxillofacial Surgery, Hebei Provincial Stomatological Hospital, No. 383, East Zhongshan Road, Shijiazhuang 050000, Hebei, China

Corresponding author: Kaicheng Yang, e-mail: cuizifeng358@foxmail.com

\section{INTRODUCTION}

Oral cancer ranks sixth among the most common cancers in the world ${ }^{1}$. Oral squamous cell carcinoma (OSCC) accounts for about $90 \%\left(\right.$ ref. $^{2}$ ), and it has the most serious impact on the quality of life. Previous studies have shown that long-term adverse stimulus factors such as tobacco, alcohol and betel nut are the causes of OSCC (ref. ${ }^{3}$ ). Although various treatment methods such as radiotherapy, chemotherapy, surgery or targeted therapy have been continuously developed in recent years, the prognosis of OSCC patients has not improved as expected in the past few years. Neck lymph node metastasis or high recurrence rate causes the 5-year survival rate of OSCC patients to be only $40 \%-50 \%\left(\right.$ ref. $\left.^{4}\right)$. Therefore, it is necessary to further explore the underlying pathogenesis of OSCC.

Tumor molecular biology has become a research hotspot in recent years, which provides the possibility to explore the pathogenesis of OSCC. For example, SCD1 played an independent prognostic role in OSCC and could be used as a candidate for targeted cancer therapy ${ }^{5}$. The length of the microRNA (miRNA/miR) is about 20 to 25 nucleotides, which have key regulatory functions in the occurrence or development of cancer, and can be involved in the regulation of tumor immune microenvironment $^{6}$, cell migration, invasion and epithelial interstitial transformation ${ }^{7}$. MiR-770 promoted the migration and invasion of OSCC by regulating the Sirt7/Smad4 pathway ${ }^{8}$. MiR-487a-3p was found to inhibit the growth and invasion of OSCC by regulating the expression of PPM1A, thereby playing a tumor suppressor effect ${ }^{9}$. However, there are few comprehensive analyses on the miRNA-mRNA regulatory network in OSCC.

In this study, we analyzed the differentially expressed miRNAs (DEMs) and differentially expressed genes (DEGs) of OSCC. GO and KEGG enrichment analysis 


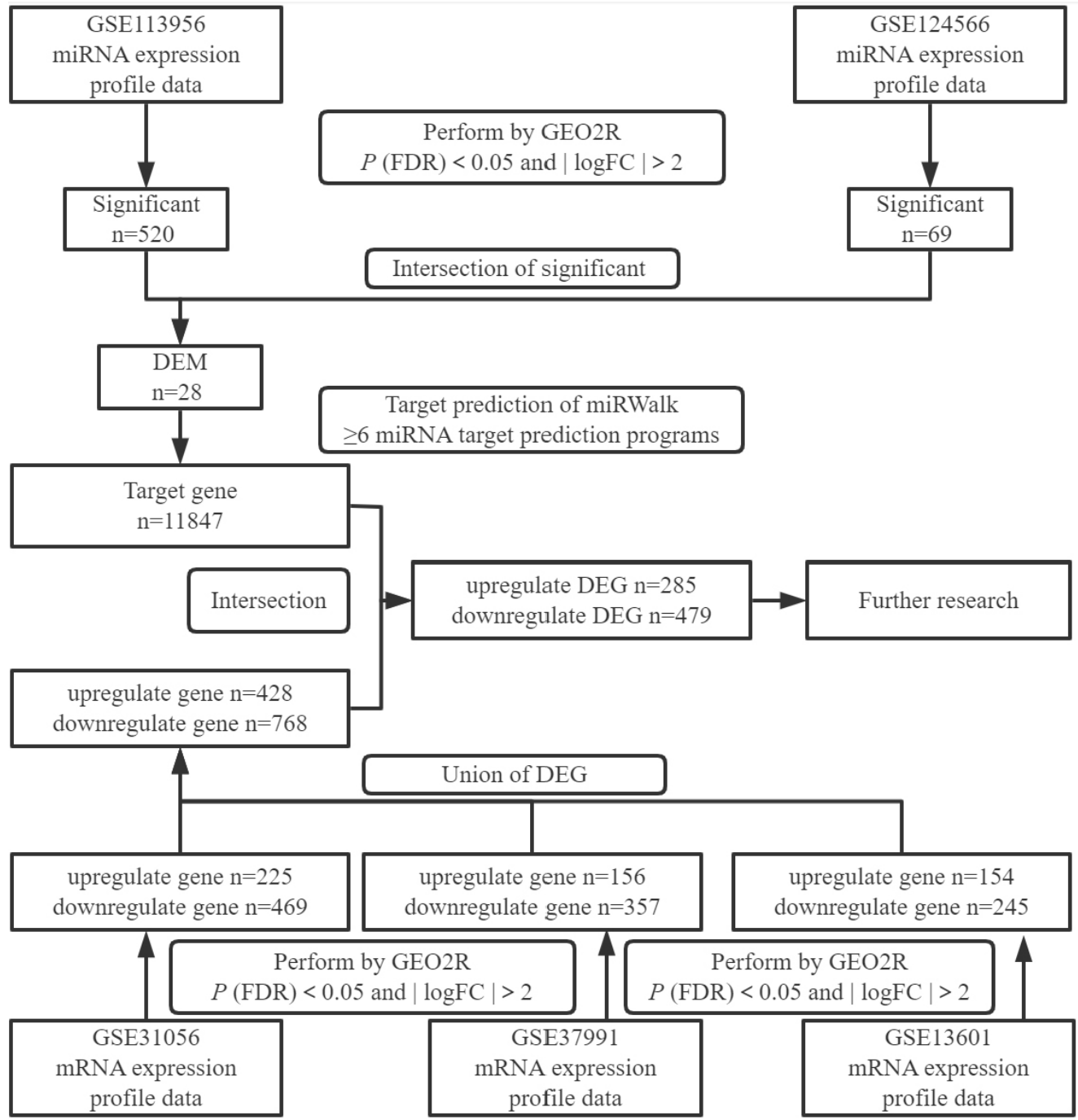

Fig. 1. Detailed screening process and conditions of DEMs and DEGs in OSCC.

were performed on DEGs, and then protein-protein interaction network (PPI network) and miRNA-mRNA regulatory network were established. Finally, the hub genes were screened out. The relationship between hub genes and clinical data were analyzed to reveal the regulatory mechanism of OSCC, explored the main pathways and processes involved, and provided a theoretical basis for the diagnosis and treatment of OSCC.

\section{MATERIALS AND METHODS}

\section{Microarray data collection}

The GSE113956, GSE124566 miRNA expression data and GSE31056, GSE37991, GSE13601 mRNA expression data were obtained from GEO database (http://www.ncbi. nlm.nih.gov/geo/) (ref. $\left.{ }^{10}\right)$. The control and corresponding OSCC samples were screened from the data for further analysis (Table 1).

Table 1. The basic information of miRNA and mRNA expression microarrays in OSCC.

\begin{tabular}{lllcc}
\hline MiRNA/Gene & GEO ID & Platform & Case: Control & Sample type \\
\hline miRNA & GSE113956 & $\begin{array}{l}\text { GPL18058 Exiqon miRCURY LNA microRNA array_7th } \\
\text { generation }\end{array}$ & $25: 15$ & OSCC \\
miRNA & GSE124566 & $\begin{array}{l}\text { GPL18402 Agilent-046064 Unrestricted_Human_miRNA_ } \\
\text { V19.0_Microarray }\end{array}$ & $10: 10$ & OSCC \\
mRNA & GSE31056 & $\begin{array}{l}\text { GPL10526 Affymetrix GeneChip Human Genome HG-U133 } \\
\text { Plus 2 Array }\end{array}$ & $23: 73$ & OSCC \\
mRNA & GSE37991 & GPL6883 Illumina HumanRef-8 v3.0 expression beadchip & $40: 40$ & OSCC \\
mRNA & GSE13601 & GPL8300 Affymetrix Human Genome U95 Version 2 Array & $37: 20$ & OSCC \\
\hline
\end{tabular}




\section{Screening of DEMs and DEGs}

The original data in the miRNA and mRNA expression data were analyzed by the GEO2R online tool (http:// www.ncbi.nlm.nih.gov/geo/geo2r/) for differential expression analysis between the control and the corresponding OSCC. MiRWalk 2.0 (http://zmf.umm.uni-heidelberg.de/ apps/zmf/mirwalk2/index.html) can provide 12 kinds of miRNA target interaction prediction and verification programs ${ }^{11}$. DEMs were submitted in the MiRWalk 2.0 database to obtain target genes that interact with miRNAs. Furthermore, it intersected with the screened mRNA to obtain the overlapping DEGs for further analysis. Fig. 1 showed the detailed screening process and conditions of DEMs and DEGs.

\section{GO and KEGG enrichment analysis}

DAVID biological information resource database (https://david.ncifcrf.gov/) can be used for gene function classification and annotation ${ }^{12}$. The biological process (BP), molecular function (MF), cell component (CC) annotation and KEGG pathway enrichment analysis were performed through the DAVID database. Data processing used R language clusterProfiler, org.Hs.eg.db, enrichplot and GOplot packages. The results were statistically significant with $P($ FDR $)<0.05$.

\section{Establishment of PPI network and preliminary screening of the hub genes}

The basic unit of interaction in STRING (https:// string-db.org/) (ref. $\left.{ }^{13}\right)$ is "functional combination", the link between two proteins, which can be used to build a PPI network. The DEGs PPI network with a comprehensive score $\geq 0.4$ was established through STRING, then imported the data into Cytoscape (version 3.6.1) software for further screening. According to the order of degree, the top 10 up-regulated genes and the top 10 down-regulated genes were selected as the preliminary hub genes.

\section{Identification of the hub genes}

The gene expression data of tumor and normal samples in OSCC patients were retrieved from the TCGA database (https://www.cancer.gov/about-nci/organiza-
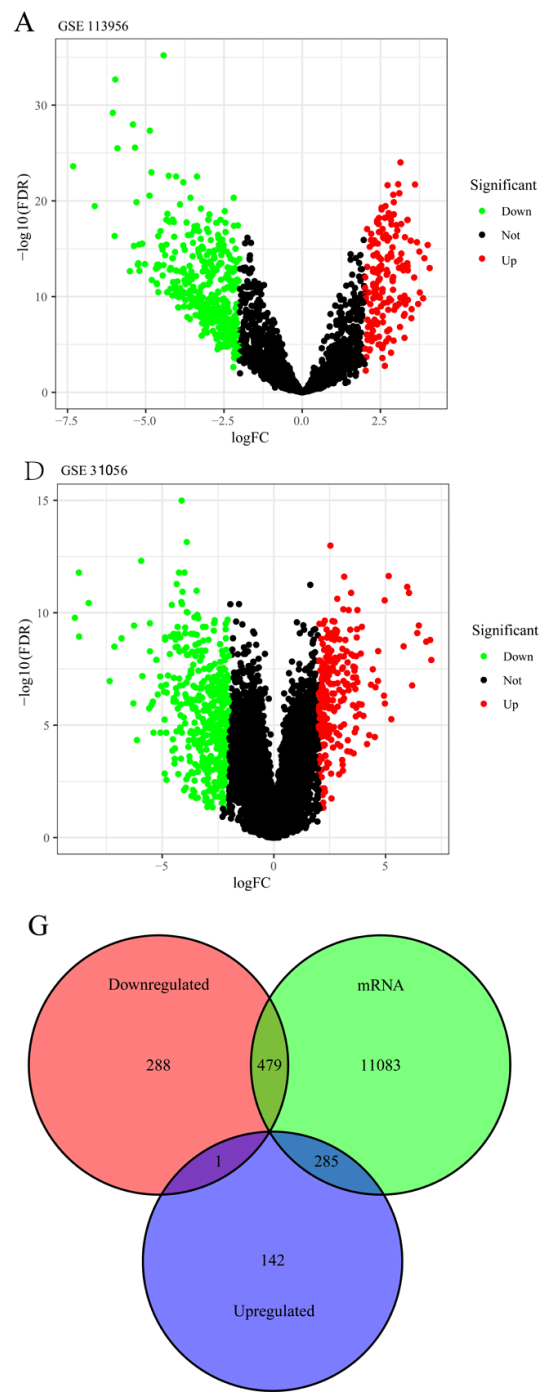
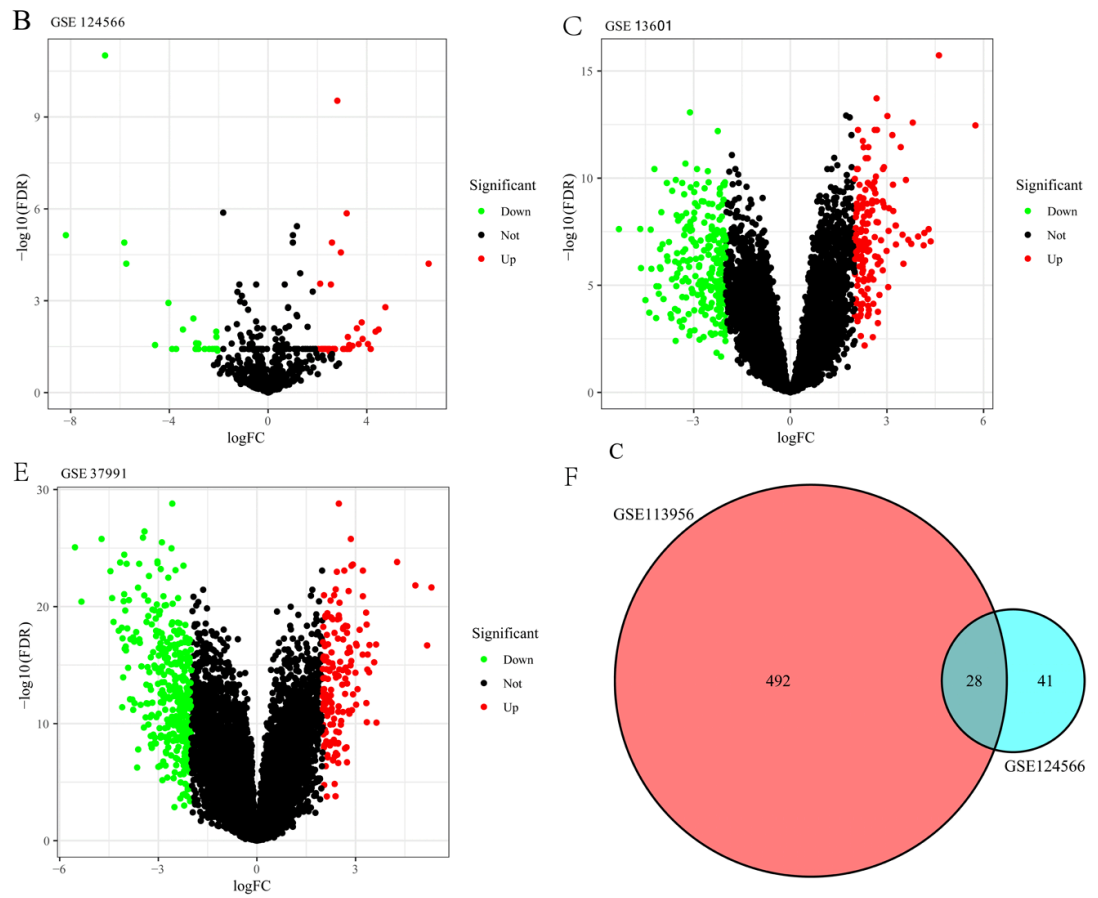

F

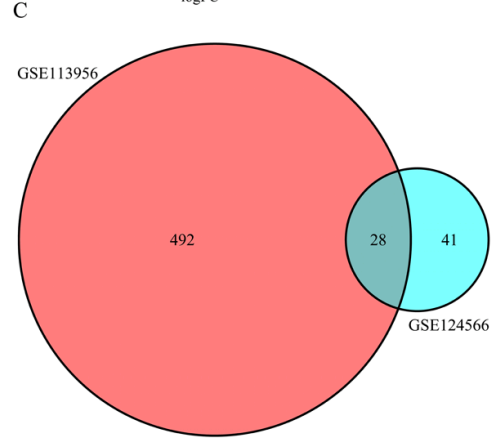

Fig. 2. Screening for differentially expressed miRNAs (DEMs) and genes (DEGs). Expression volcano map of GSE113956(A), GSE124566(B), GSE13601(C), GSE31056(D) and GSE37991(E). Red dots represented up-regulation, green dots represented down-regulation, and black dots represented no significant changes in expression levels. $P(\mathrm{FDR})<0.05$ and $|\log \mathrm{FC}|>2$. (F) 28 DEMs were screened out. (G) 285 up-regulated DEGs and 479 down-regulated DEGs were screened out. 
Table 2. Enrichment analysis of GO in DEGs.

\begin{tabular}{llcc}
\hline GO & & & \\
up-regulated & Term & $P(\mathrm{FDR})$ & Count \\
\hline BP & extracellular matrix organization & $4.13 \mathrm{E}-32$ & 52 \\
BP & extracellular structure organization & $4.13 \mathrm{E}-32$ & 52 \\
CC & collagen-containing extracellular matrix & $4.33 \mathrm{E}-20$ & 41 \\
MF & extracellular matrix structural constituent & $1.21 \mathrm{E}-16$ & 26 \\
CC & endoplasmic reticulum lumen & $2.10 \mathrm{E}-11$ & 26 \\
BP & cell junction assembly & $9.99 \mathrm{E}-07$ & 25 \\
\hline GO & & & \\
down-regulated & Term & $P(\mathrm{FDR})$ & Count \\
\hline BP & muscle system process & $2.60 \mathrm{E}-19$ & 54 \\
CC & contractile fiber & $1.66 \mathrm{E}-25$ & 45 \\
CC & myofibril & $1.66 \mathrm{E}-25$ & 44 \\
BP & muscle contraction & $1.51 \mathrm{E}-15$ & 43 \\
CC & sarcomere & $3.11 \mathrm{E}-24$ & 41 \\
MF & actin binding & $1.28 \mathrm{E}-09$ & 39 \\
\hline
\end{tabular}

Table 3. Enrichment analysis of KEGG pathways in DEGs.

\begin{tabular}{llll}
\hline KEGG pathways: & Term & $P(\mathrm{FDR})$ & Count \\
& & & \\
\cline { 2 - 4 } & pathways in cancer & $4.33 \mathrm{E}-04$ & 22 \\
& PI3K-Akt signaling pathway & $7.94 \mathrm{E}-05$ & 22 \\
& focal adhesion & $1.59 \mathrm{E}-07$ & 21 \\
& ECM-receptor interaction & $1.60 \mathrm{E}-14$ & 21 \\
& amoebiasis & $4.26 \mathrm{E}-06$ & 14 \\
& protein digestion and absorption & $4.26 \mathrm{E}-06$ & 13 \\
& small cell lung cancer & $1.34 \mathrm{E}-04$ & 11 \\
& cell cycle & $4.99 \mathrm{E}-02$ & 9 \\
& arrhythmogenic right ventricular cardiomyopathy & $6.03 \mathrm{E}-03$ & 8 \\
& (ARVC) & & \\
\hline KEGG pathways: & Term & $P(\mathrm{FDR})$ & Count \\
& & & \\
\cline { 2 - 4 } down-regulated & adrenergic signaling in cardiomyocytes & $5.19 \mathrm{E}-03$ & 15 \\
& AMPK signaling pathway & $7.25 \mathrm{E}-03$ & 13 \\
& hypertrophic cardiomyopathy (HCM) & $5.27 \mathrm{E}-03$ & 11 \\
& dilated cardiomyopathy & $6.67 \mathrm{E}-03$ & 11 \\
& insulin resistance & $2.63 \mathrm{E}-02$ & 11 \\
& adipocytokine signaling pathway & $2.63 \mathrm{E}-02$ & 9 \\
\hline
\end{tabular}

Count: the number of enriched genes, KEGG: Kyoto Encyclopedia of Genes and Genomes. $P(\mathrm{FDR})<0.05$.

tion/ccg/research/structural-genomics/tcga), including 32 normal controls and 330 cases of OSCC. The hub genes were verified by differential expression analysis. The data was processed through the $\mathrm{R}$ software (version 4.0.3) limma package with $P($ FDR $)<0.05$ and $|\log \mathrm{FC}|>1$ as the screening conditions.

\section{Establishment of the miRNA-mRNA regulatory network}

Application of miRTarBase (http://mirtarbase.cuhk. edu.cn/php/index.php) (ref. ${ }^{14}$ ) and TargetScan (http:// www.targetscan.org/vert_72/) (ref..$\left.^{15}\right)$ predicted the target genes of DEMs and got the intersection. When these intersection genes and the hub genes shared a common target mRNA there may be similar regulatory pathways, to establish miRNA-mRNA regulatory networks.

\section{Clinical data analysis of the hub genes}

A total of 328 patients with OSCC were screened in the TCGA database to analyze the relationship between the expression level of the hub genes and overall survival (OS) and tumor differentiation. Unpaired Student's t-test or one-way ANOVA test was used to compare normally distributed data. Non-normally distributed data were performed using the Mann-Whitney U test or Kruskal-Wallis test. Uni- and multivariate analyses were performed using Cox proportional hazard models with the stepwise meth- 
od. Survival differences were evaluated using the KaplanMeier estimate with the log-rank test. All statistical tests and visual analyses were performed using $\mathrm{R}$ software or GraphPad Prism (version 6.0).

\section{RESULTS}

\section{Screening of DEM and DEG}

From the expression data of GSE113956 (Fig. 2A) and GSE124566 (Fig. 2B), 520 and 69 miRNAs were screened respectively, then 28 intersecting DEMs were screened from the two sets of miRNA data (Fig. 2F), including hsa-miR-10a-5p, hsa-miR-142-3p, hsa-miR-1425p, hsa-miR-146b-5p, hsa-miR-181c-5p, hsa-miR-181d, hsa-miR-193b-5p, hsa-miR-196a-5p, hsa-miR-196b-5p, hsa-miR-203a, hsa-miR-218-5p, hsa-miR-22-5p, hsa-miR29b-1-5p, hsa-miR-29c- 5p, hsa-miR-3188, hsa-miR-338-3p, hsa-miR-342-5p, hsa-miR-375, hsa-miR-378a-5p, hsa-miR382-5p, hsa-miR-409-3p, hsa-miR-4419a, hsa-miR-4647, hsa-miR-4778-5p, hsa-miR-513b, hsa-miR-513c-5p, hsamiR-625-5p and hsa-miR-744-5p. In the expression data of GSE13601 (Fig. 2C), GSE31056 (Fig. 2D) and GSE37991 (Fig. 2E), a total of 428 up-regulated genes and 768 down-regulated genes were screened. Next, the genes that intersected with 11,847 target genes of 28 DEMs predicted by MiRWalk 2.0 were selected (Fig. $2 \mathrm{G}$ ). Finally, there were 285 up-regulated DEGs and 479 down-regulated DEGs for further study.

\section{Enrichment analysis of GO and KEGG pathways}

The GO function annotations of 285 DEGs up-regulated showed that the main MF was the extracellular matrix structural constituent, the main BP was involved in the extracellular matrix organization, extracellular structure organization and cell junction assembly, the main $\mathrm{CC}$ was collagen-containing extracellular matrix and endoplasmic reticulum lumen. The GO functional annotations of the down-regulated 479 DEGs showed that the main MF was actin binding, the main BP was involved in the muscle system process and muscle contraction, the main CC was contractile fiber, myofibril and sarcomeres (Table 2).

The KEGG pathway enrichment analysis showed that the up-regulated DEGs were mainly enriched in cancerrelated pathways and PI3K-Akt signaling pathway, the down-regulated DEGs were mainly enriched in the adrenaline signaling and AMPK signaling pathway (Table 3 ).

In $\mathrm{GO}$ analysis, the top 6 terms enriched by up-regulated DEGs and the top 6 terms enriched by down-regulated DEGs. Count: the number of enriched genes, GO: Gene ontology, BP: biological process, MF: molecular function, $\mathrm{CC}$ : cell component. $P(\mathrm{FDR})<0.05$.

\section{Construction of PPI network and preliminary screening of hub genes}

The PPI network of DEGs was established in STRING. The up-regulated network includes 235 nodes and 1,599 edges, and the down-regulated network includes
Table 4. The degree of genes in the protein interaction network of DEGs.

\begin{tabular}{lll}
\hline Up-regulated DEGs & ID & Degree \\
\cline { 2 - 3 } & FN1 & 76 \\
CDH1 & 58 \\
CD44 & 52 \\
COL1A1 & 46 \\
COL1A2 & 39 \\
AURKA & 39 \\
CCNB1 & 39 \\
CCNA2 & 38 \\
SPP1 & 38 \\
CDC6 & 37 \\
\hline Down-regulated DEGs & ID & Degree \\
\cline { 2 - 3 } & ACTN2 & 47 \\
TTN & 45 \\
IGF1 & 36 \\
CAV3 & 36 \\
MYL2 & 34 \\
DMD & 34 \\
LDB3 & 34 \\
CSRP3 & 34 \\
ACTA1 & 33 \\
PPARG & 31 \\
\hline
\end{tabular}

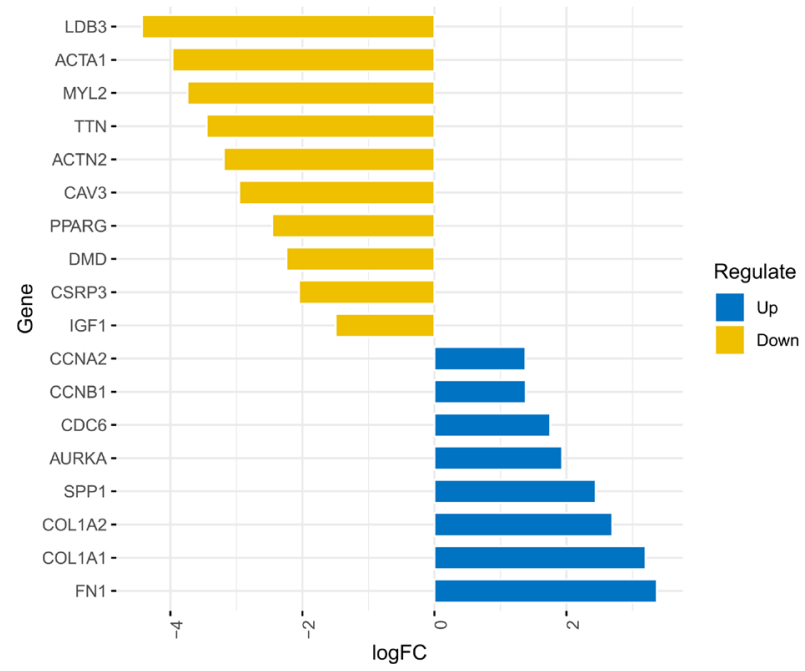

Fig. 3. TCGA database identified the differential expression of hub genes. The figure showed hub genes that meet FDR $<0.05$ and $|\log \mathrm{FC}|>1$. Blue represented up-regulated hub gens, and yellow represented down-regulated hub gens.

431 nodes and 1,454 edges. The edge represented the interaction between proteins. After the data were imported into Cytoscape, "Degree" was applied to represent the edge information of genes, and the top 10 genes with upregulation and down-regulation of degrees were selected as the preliminary hub genes of OSCC. The up-regulated DEGs included FN1, CDH1, CD44, COL1A1, COL1A2, AURKA, CCNB1, CCNA2, SPP1, CDC6, and the down- 
regulated DEGs included ACTN2, TTN, IGF1, CAV3, MYL2, DMD, LDB3, CSRP3, ACTA1, PPARG. (Table 4)

In protein interaction network of DEGs, the top 10 genes of up-regulation degrees and the top 10 genes of down-regulation degrees. Degree: the number of interaction between proteins.

\section{Hub genes verification in TCGA database}

According to the differential expression analysis of the 20 preliminary hub genes expression levels in 330 OSCC tumors and 32 normal control samples in the TCGA database, it was found that the expression levels of CDH1 and CD44 were not different and the expression trend of the remaining 8 up-regulated hub genes and 10 downregulated hub genes were consistent with the results of the GEO database (Fig. 3).

\section{Construction of MiRNA-mRNA Regulatory Network}

In order to more clearly show the regulatory relationship between 28 DEMs and 18 hub genes, a miRNAmRNA regulatory network was established (Fig. 4). Among the 18 hub genes, there were no hsa-miR-146b5p, hsa-miR-181d, hsa-miR-196b-5p, hsa-miR-29c-5p, hsamiR-375, hsa-miR-409- 3p, hsa-miR-4419a, hsa-miR-4647 and hsa-miR-744-5p possible targets. MYL2, CSRP3 and ACTA1 also did not show possible regulated relationships. Hsa-miR-513b was the most regulated DEM, targeting 8 hub genes, including the up-regulated COL1A1, COL1A2, CCNB1, CCNA2, CDC6, and the down-regulated TTN, IGF1, DMD. The COL1A1 target was predicted the most, as common target of hsa-miR-193b-5p, hsa-miR-196a-5p, hsa-miR-218-5p, hsa-miR-29b-1-5p, hsa-miR-338-3p, hsa-

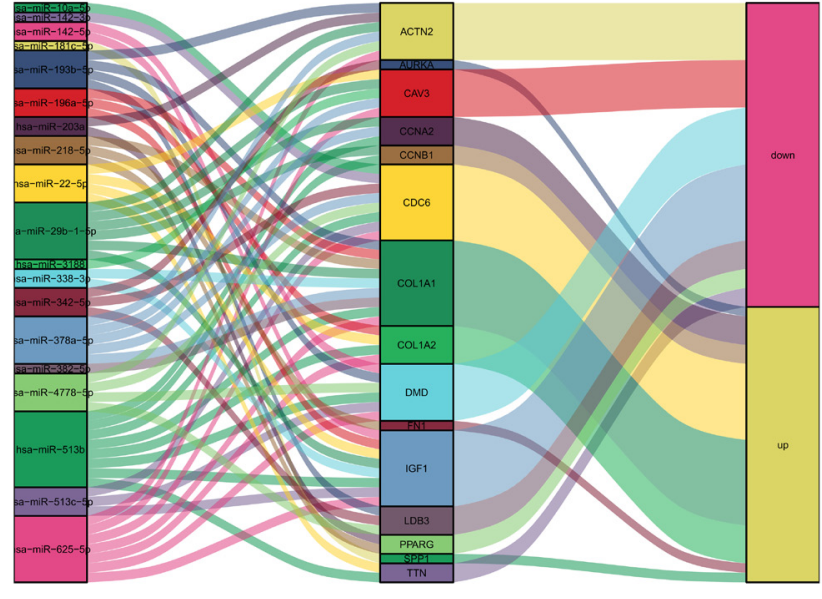

miRNA

mRNA

Type

Fig. 4. MiRNA-mRNA regulatory network in OSCC. The first column represented miRNAs, the second column represented mRNAs, the third column represented the expression trend of mRNAs, and the connecting lines between them represented regulatory relationships.

miR-378a-5p, hsa-miR-382-5p, hsa-miR-513b and hsa-miR625-5p.

\section{Analysis of the correlation between hub genes and clinical data}

We used the "pheatmap" package in R to visualize the expression patterns of 15 hub genes between TCGA OSCC patients with different clinical information as a heat map (Fig. 5).

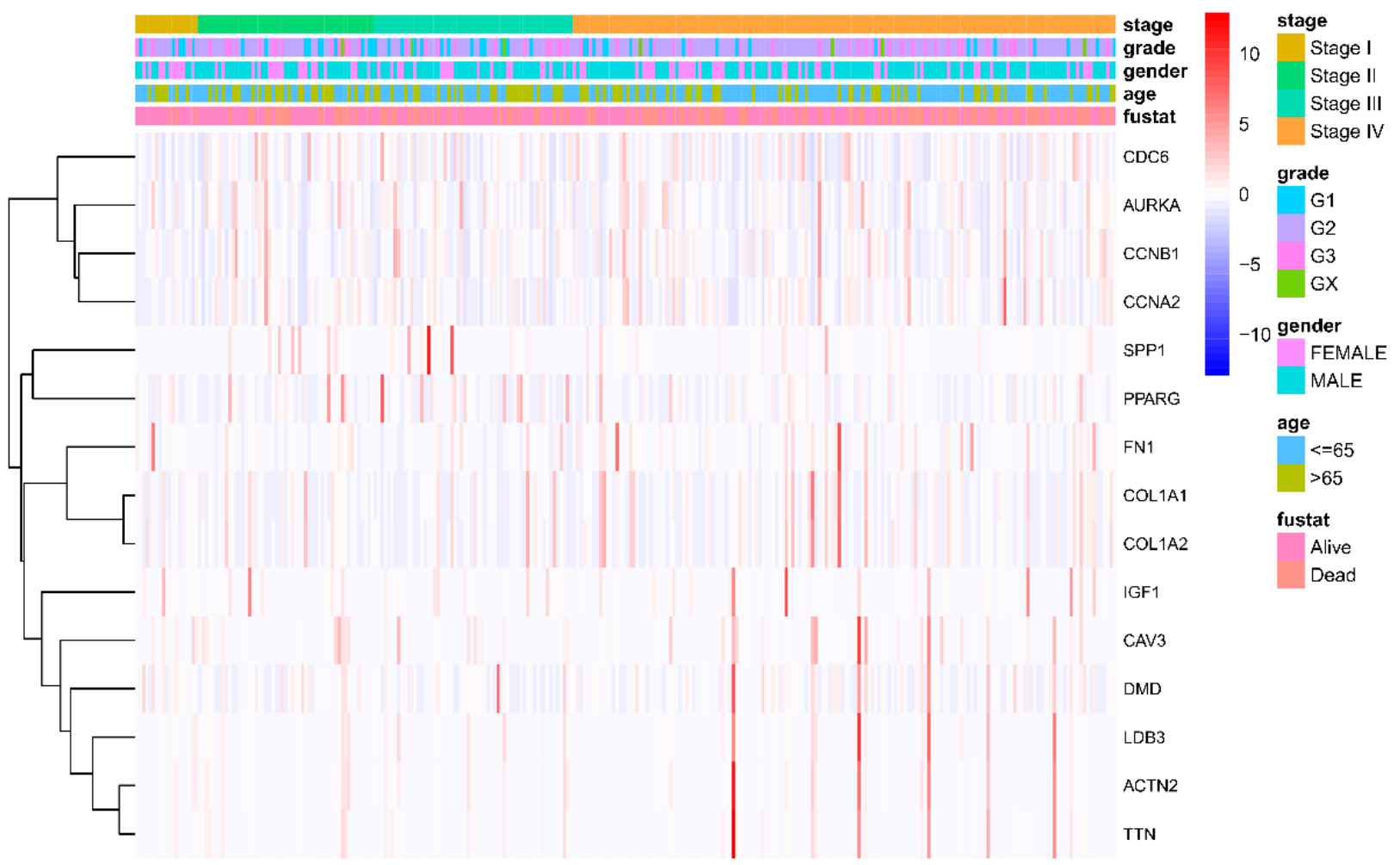

Fig. 5. Heat map shows the relative expression of 15 hub genes in TCGA OSCC patients and clinicopathological features. Grade: tumor differentiation, GX: grade cannot be assessed, G1: highly differentiated, G2: moderately differentiated, G3: poorly differentiated. 
A

\begin{tabular}{|c|c|c|c|}
\hline Characteristics & $\mathrm{HR}(95 \% \mathrm{Cl})$ & & Univariate analysis $\mathrm{P}$-value \\
\hline CDC6 & $1.309(1.048-1.634)$ & $\longmapsto$ & 0.017 \\
\hline AURKA & $1.301(1.044-1.621)$ & 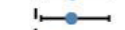 & 0.019 \\
\hline CCNB1 & $1.239(1.015-1.513)$ & 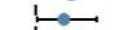 & 0.035 \\
\hline CCNA2 & $1.386(1.115-1.721)$ & - & 0.003 \\
\hline SPP1 & $1.061(0.991-1.135)$ & s. & 0.088 \\
\hline PPARG & $1.232(0.959-1.582)$ & 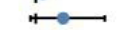 & 0.103 \\
\hline FN1 & $1.031(0.950-1.117)$ & we & 0.466 \\
\hline COL1A1 & $0.981(0.897-1.074)$ & 4 & 0.679 \\
\hline COL1A2 & $0.970(0.887-1.061)$ & 1 & 0.512 \\
\hline IGF1 & $1.754(0.866-3.555)$ & T & 0.119 \\
\hline CAV3 & $1.048(0.881-1.246)$ & $\leftrightarrow$ & 0.599 \\
\hline DMD & $0.918(0.697-1.207)$ & - & 0.539 \\
\hline LDB3 & $1.081(0.955-1.224)$ & Ien & 0.216 \\
\hline ACTN2 & $1.062(0.988-1.141)$ & tat & 0.102 \\
\hline TTN & $1.113(0.977-1.269)$ & tor & 0.108 \\
\hline
\end{tabular}

$\mathrm{C}$
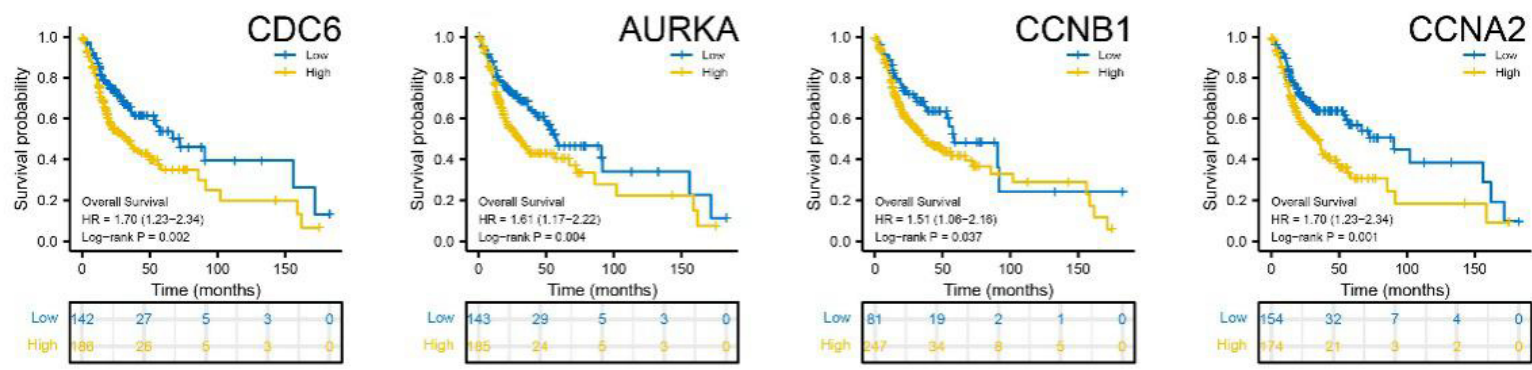

D
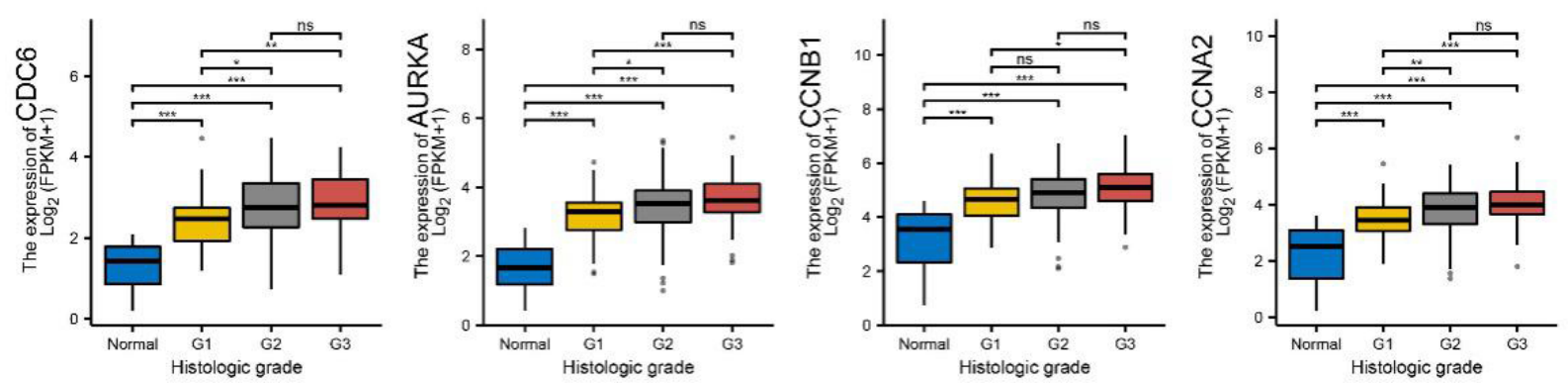

Fig. 6. Screening the hub genes associated with the prognosis of OSCC patients.

(A) Univariate cox regression showed the relationship between 15 hub genes and patient prognosis. $P<0.05$. (B) Stepwise multivariate cox regression excluded genes that were not significantly associated with the patient's prognosis. (C) Kaplan-Meier plot and the log-rank test showed the survival rate of patients with high or low expression of these genes. $P<0.05$. (D) Box plots showed the expression of these four genes in different OSCC pathological grades. G1: highly differentiated, G2: moderately differentiated, G3: poorly differentiated. $* * P<0.001, * * P<0.01, * P<0.05$.

In order to further determine the relationship between the prognosis of these 15 hub genes and OSCC patients, we used the univariate cox regression method at first. The results were given in Fig. $6 \mathrm{~A}$, where the hazard ratios (HRs) of genes were $<1$, indicating that their overexpression was associated with longer OS, but the other gene with HR >1 meant the opposite. Following that, we utilized stepwise multivariate cox regression to exclude genes that were not significantly associated with the patient's prognosis. As a result, only four genes (CDC6, AURKA, CCNB1 and CCNA2 ) were retained (Fig. 6B). We analyzed the difference in survival between patients with high and low expression of these genes using a Kaplan-Meier plot and the log-rank test. Fig. 6C showed that patients with high expression of these genes had a worse chance of survival. Furthermore, we used box plots to show the expression of these four genes in different OSCC tumor differentiation. As demonstrated in Fig. 6D, the poorer the tumor differentiation of OSCC, the higher the relative expression levels of these four genes. The results were statistically significant at $P<0.05$.

\section{DISCUSSION}

OSCC is one of the main types of head and neck squamous cell carcinoma, and its incidence is still increasing. In 2018 alone, there were about 354,000 new cases, and about 177,000 patients have died, indicating that the survival rate is still low ${ }^{16}$. Although great progress has been made in early screening and personalized treatment of OSCC in recent years, OSCC is still one of the diseases that has attracted much attention because of its serious impact on the quality of life of patients such as eating and language ${ }^{17}$. The molecular mechanisms involved in the progress of OSCC are still unclear. Therefore, the study 
of OSCC needs to clarify the pathogenesis and screen targets related to diagnosis, treatment and prognosis.

In this study, a total of 28 DEMs were screened out from the miRNA expression data of GSE113956 and GSE124566, most of which had been confirmed to be involved in the pathogenesis of OSCC. For example, the hsa-miR-142-3p inhibited FoxO1 activity through the activation of the PI3K/ Akt signaling pathway, which was closely related to the pathological grade and clinical stage of OSCC (ref. ${ }^{18}$ ). Hsa-miR-142-5p was targeted to up-regulate IGF2BP3 to facilitate the invasion and metastasis of OSCC $\left(\right.$ ref. $^{19}$ ). K-ras was the target of hsa-miR-181d, and the overexpression of K-ras effectively reversed the inhibitory effect of hsa-miR-181d on the proliferation and apoptosis of OSCC (ref. ${ }^{20}$ ). Hsa-miR-196a-5p induced apoptosis avoidance and therapeutic resistance in OSCC cells by targeting BIRC3 (ref. ${ }^{21}$ ). Hsa-miR-218-5p inhibited OSCC cell invasion by targeting the CD44-ROCK signaling pathway ${ }^{22}$. The oncogenic activity of hsa-miR-29b-1$5 \mathrm{p}$ could induce the epithelial-mesenchymal transition of OSCC (ref. ${ }^{23}$ ). Hsa-miR-29c-5p inhibited cell proliferation and migration of head and neck cancer by targeting TMEM98 (ref. ${ }^{24}$ ). The elucidation of the pathogenesis of these DEMs in OSCC provides potential biomarkers for diagnosis and treatment.

In the DEGs enrichment analysis, it was found that the up-regulated DEGs were mainly enriched in extracellular matrix (ECM), cancer-related pathways and PI3KAkt signaling pathways. ECM was closely associated with tumor metastasis, and successful metastasis of cancer cells required inhibition of anoikis, a cell death program caused by loss of attachment to ECM (ref. ${ }^{25}$ ). ECM separation could also regulate the metabolism of cancer cells in a variety of different cell types, and interfering with the metabolism in ECM-separated cells might be an effective treatment for selectively inhibiting tumor progression ${ }^{26}$. The PI3K-Akt signaling pathway regulated many basic cellular processes, such as metabolism, autophagy, and protein synthesis, and its activity could lead to malignant transformation of cells. Abnormal activation of the PI3K/ Akt pathway has been found in about $90 \%$ of lung adenocarcinoma and $40 \%$ of squamous cell carcinoma ${ }^{27}$. The down-regulated DEGs were mainly concentrated in musculature processes and AMPK signaling pathways. AMPK was a downstream target of the tumor suppressor LKB1, which might play a role in inhibiting tumorigenesis, but recent studies have shown that AMPK could inhibit or promote cancer according to the situation. AMPK could inhibit abnormal growth before the occurrence of cancer, but after cancer occurs, AMPK could support the survival of cancer cells by regulating the growth rate of cancer cells to match the energy supply, thereby promoting genome stability ${ }^{28}$.

By targeting mRNA, miRNA was involved in the regulation of various cellular processes such as cell proliferation, apoptosis and signal transduction, and played an important role in the development of tumors ${ }^{29}$. The abnormal expression of miRNA in OSCC caused the degradation or translation inhibition of the regulated mRNA, and then played the role of tumor suppressor gene or oncogene ${ }^{30}$. Therefore, the establishment of the miRNAmRNA regulatory network was particularly important for the study of the pathogenesis of OSCC. After screening and verification by the PPI network and TCGA, it was found that 18 hub genes might play a key role in the pathogenesis of OSCC. For example, FN1 belonged to the extracellular matrix glycoprotein family, existed in a variety of cell types, participated in cell adhesion, migration and other processes. Its expression was up-regulated through the NF-kB pathway to inhibit tumor cell apoptosis and lead to cell migration ${ }^{31}$. It was considered a potential biomarker for tongue and floor squamous cell carcinoma in OSCC. The construction of miRNA-mRNA regulatory network showed that COL1A1 was the most predicted target, including hsa-miR-193b-5p, hsa-miR-196a-5p, hsa-miR-218-5p, hsa-miR-29b-1-5p , hsa-miR-338-3p, hsamiR-378a-5p, hsa-miR-382-5p, hsa-miR-513b and hsa-miR625-5p. COL1A1, a member of the collagen family, was widely distributed in ECM and could regulate intercellular adhesion and differentiation. Its up-regulated expression could promote the proliferation and migration of OSCC cells $^{32}$. Hsa-miR-513b had the most regulation, targeting 8 hub genes, including up-regulated COL1A1, COL1A2, CCNB1, CCNA2, CDC6, down-regulated TTN, IGF1, DMD. Significantly, the regulatory relationship of hsamiR-513b/ CDC6, CCNB1, CCNA2 and the regulatory relationship of hsa-miR-342-5p /AURKA were not only verified in the miRNA-mRNA regulatory network, but also related to overall survival and tumor differentiation. This indicated that they were involved in the cellular regulatory process and had potential clinical application value.

Hsa-miR-513b and hsa-miR-342 was abnormally expressed in a variety of cancers, including liver cancer, colon cancer and ovarian carcinoma ${ }^{33-36}$, but their expression in OSCC has not been clarified. CCNA2 and CCNB1 were both cell cycle proteins. CCNA2 was necessary for S-phase DNA synthesis and through the G2/M process. Its overexpression was a poor prognostic factor for oral precancerous and malignant lesions ${ }^{37}$. CCNB1 regulated maturation promoting factor (MPF). At the junction of cell $\mathrm{G} 2 / \mathrm{M}$ phase, it drove the cell cycle phase transition and initiated cell mitosis ${ }^{38}$. CCNB1 was highly expressed in tumor cells and tissues, so that MPF could still be activated when the DNA of the cells was damaged, leading to continued mitosis of the cells, and finally the formation of tumors ${ }^{39}$. Silencing of CCNB1 gene was able to inhibit proliferation, invasion and migration of human oral squamous carcinoma SCC-15 cells, and its mechanism of action might be related to the inhibition of PI3K/Akt signaling pathway activation ${ }^{40}$. AURKA kinase was one of three highly homologous serine/threonine kinase families. As a cell cycle regulating kinase, it regulated many links of mitosis. Especially during the transition from G2 to M phase, the activity of AURKA increased significantly ${ }^{41}$. AURKA could participate in many important cell signaling pathways, directly or indirectly activated a variety of oncoproteins or inactivated a variety of tumor suppressor proteins, thereby promoting the occurrence and development of tumors. It regulated EMT and apoptosis through reactive oxygen species, promoted the progression of 
OSCC, and could be used as a predictor of chemotherapy response and prognosis in patients with advanced OSCC (ref. ${ }^{42,43}$ ).CDC6 played an important role in the assembly of the pre-replication complex in the G1 phase of the cell cycle and was an essential protein for the initiation of cell DNA replication. Its up-regulated expression indicated that cells enter the proliferation cycle and enhance cell proliferation activity, while its expression was reduced or even absent in the static cells. The up-regulated expression of CDC6 in OSCC might indicate that the tumor was accompanied by lymph node metastasis or was mostly stage III or IV, which could be used to evaluate the risk of tumor recurrence or metastasis and monitor the effectiveness of treatment ${ }^{44}$.

\section{CONCLUSION}

In summary, this study screened a total of 28 DEMs and 764 DEGs consisting of 285 up-regulated and 479 down-regulated genes. We found that the regulatory relationship of hsa-miR-513b/ CDC6, CCNB1, CCNA2 and the regulatory relationship of hsa-miR-342-5p /AURKA were not only verified in the miRNA-mRNA regulatory network, but also related to overall survival and tumor differentiation. This suggests that they are involved in cellular regulatory processes and have potential clinical applications. It can be seen that revealing the miRNAmediated hub gene regulatory network in OSCC provides a molecular mechanism model for the study of pathogenesis, and also provides an effective basis for targets for diagnosis, treatment and prognosis. However, the relationship between some miRNAs or hub genes in the study and OSCC is still unclear and requires further study and verification, in order to build a more complete gene regulation network of OSCC.

Acknowledgments: We are grateful for the data shared by databases such as GEO and TCGA. We thank the reviewers for their constructive comments.

Author contributions: KY, YC: conceived and designed the study; ZC, QS: execution of study; ZC, QS: data analysis; ZC, QS, KY: discussion of results; KY, YC: wrote and critically revisedthe manuscript.

Conflicts of interest statement: The authors have no conflicts of interest to declare.

Compliance with ethical standards: The study was conducted in accordance with the Declaration of Helsinki (as revised in 2013). All data used in our study are available in public databases. Individual consent for this analysis was waived.

\section{REFERENCES}

1. Jemal A, Bray F, Center MM, Ferlay J, Ward E, Forman D. Global cancer statistics. CA Cancer J Clin 2011;61(2):69-90. doi:10.3322/caac.20107

2. Sankaranarayanan $R$, Swaminathan R, Brenner $H$, Chen K, Chia KS, Chen JG, Law SC, Ahn YO, Xiang YB, Yeole BB, Shin HR, Shanta V, Woo ZH, Martin N, Sumitsawan Y, Sriplung H, Barboza AO, Eser S,
Nene BM, Suwanrungruang K, Jayalekshmi P, Dikshit R, Wabinga $H$, Esteban DB, Laudico A, Bhurgri Y, Bah E, Al-Hamdan N. Cancer survival in Africa, Asia, and Central America: a population-based study. Lancet Oncol 2010;11(2):165-73. doi: 10.1016/S1470-2045(09)703353

3. Warnakulasuriya S. Global epidemiology of oral and oropharyngeal cancer. Oral Oncol 2009;45(4-5):309-16. doi: 10.1016/j.oraloncology.2008.06.002

4. Coleman MP, Quaresma M, Berrino F, Lutz JM, De Angelis R, Capocaccia R, Baili P, Rachet B, Gatta G, Hakulinen T, Micheli A, Sant M, Weir HK, Elwood JM, Tsukuma H, Koifman S, E Silva GA, Francisci $\mathrm{S}$, Santaquilani M, Verdecchia A, Storm HH, Young JL; CONCORD Working Group. Cancer survival in five continents: a worldwide population-based study (CONCORD). Lancet Oncol 2008;9(8):73056. doi: 10.1016/S1470-2045(08)70179-7

5. Schminke B, Shomroni O, Salinas G, Bremmer F, Kauffmann P, Schliephake H, Oyelami F, Rahat MA, Brockmeyer P. Prognostic factor identification by screening changes in differentially expressed genes in oral squamous cell carcinoma. Oral Dis 2021 Apr 19. [Epub ahead of print] doi: 10.1111/odi.13879

6. Mondal P, Kaur B, Natesh J, Meeran SM. The emerging role of miRNA in the perturbation of tumor immune microenvironment in chemoresistance: Therapeutic implications. Semin Cell Dev Biol $2021 \mathrm{Apr}$ 14. [Epub ahead of print] doi: 10.1016/j.semcdb.2021.04.001

7. Wang Y, Hao W, Wang H. miR-557 suppressed the malignant behaviours of osteosarcoma cells by reducing HOXB9 and deactivating the EMT process. Artif Cells Nanomed Biotechnol 2021;49(1):230-39. doi: 10.1080/21691401.2021.1890100

8. Jia B, Zhang S, Wu S, Zhu Q, Li W. MiR-770 promotes oral squamous cell carcinoma migration and invasion by regulating the Sirt7/Smad4 pathway. IUBMB Life 2021;73(1):264-72. doi: 10.1002/iub.2426

9. Wang L, Ge S, Zhou F. MicroRNA-487a-3p inhibits the growth and invasiveness of oral squamous cell carcinoma by targeting PPM1A. Bioengineered 2021;12(1):937-47. doi: 10.1080/21655979.2021.1884396

10. Barrett T, Wilhite SE, Ledoux P, Evangelista C, Kim IF, Tomashevsky M, Marshall KA, Phillippy KH, Sherman PM, Holko M, Yefanov A, Lee $\mathrm{H}$, Zhang N, Robertson CL, Serova N, Davis S, Soboleva A. NCBI GEO: archive for functional genomics data sets--update. Nucleic Acids Res 2013;41(Database issue):D991-5. doi: 10.1093/nar/gks1193

11. Dweep H, Sticht C, Pandey P, Gretz N. miRWalk--database: prediction of possible miRNA binding sites by "walking" the genes of three genomes. J Biomed Inform 2011;44(5):839-47. doi: 10.1016/j. jbi.2011.05.002

12. Huang da W, Sherman BT, Lempicki RA. Systematic and integrative analysis of large gene lists using DAVID bioinformatics resources. Nat Protoc 2009;4(1):44-57. doi: 10.1038/nprot.2008.211

13. Szklarczyk D, Gable AL, Lyon D, Junge A, Wyder S, Huerta-Cepas J, Simonovic M, Doncheva NT, Morris JH, Bork P, Jensen LJ, Mering CV. STRING v11: protein-protein association networks with increased coverage, supporting functional discovery in genome-wide experimental datasets. Nucleic Acids Res 2019;47(D1):D607-D613. doi: 10.1093/nar/gky1131

14. Huang HY, Lin YC, Li J, Huang KY, Shrestha S, Hong HC, Tang Y, Chen YG, Jin CN, Yu Y, Xu JT, Li YM, Cai XX, Zhou ZY, Chen XH, Pei YY, Hu L, Su JJ, Cui SD, Wang F, Xie YY, Ding SY, Luo MF, Chou CH, Chang NW, Chen KW, Cheng YH, Wan XH, Hsu WL, Lee TY, Wei FX, Huang HD. miRTarBase 2020: updates to the experimentally validated microRNA-target interaction database. Nucleic Acids Res 2020;48(D1):D148-D154. doi: 10.1093/nar/gkz896

15. Agarwal V, Bell GW, Nam JW, Bartel DP. Predicting effective microRNA target sites in mammalian mRNAs. Elife 2015;4:e05005. doi: 10.7554/ eLife.05005

16. Bray F, Ferlay J, Soerjomataram I, Siegel RL, Torre LA, Jemal A. Global cancer statistics 2018: GLOBOCAN estimates of incidence and mortality worldwide for 36 cancers in 185 countries. CA Cancer J Clin 2018;68(6):394-424. doi: 10.3322/caac.21492

17. Jiang $S$, Dong Y. Human papillomavirus and oral squamous cell carcinoma: A review of HPV-positive oral squamous cell carcinoma and possible strategies for future. Curr Probl Cancer 2017;41(5):323-27. doi: 10.1016/j.currproblcancer.2017.02.006

18. Zhang Yun, Li Shengmei, Zhang Yuan, Chang Qunan. Relationship between expression of transcription factor FoxO1 and miR-142$3 p$ in oral squamous cell carcinoma and its significance. J West 
Med 2020;32(09):1333-1336+1341. doi:10.3969/j.issn.16723511.2020.09.016

19. Liu J, Jiang X, Zou A, Mai Z, Huang Z, Sun L, Zhao J. circlGHG-Induced Epithelial-to-Mesenchymal Transition Promotes Oral Squamous Cell Carcinoma Progression via miR-142-5p/IGF2BP3 Signaling. Cancer Res 2021;81(2):344-55. doi: 10.1158/0008-5472.CAN-20-0554

20. Shi Y, Li Y, Wu W, Xu B, Ju J. MiR-181d functions as a potential tumor suppressor in oral squamous cell carcinoma by targeting K-ras. Int J Clin Exp Pathol 2017;10(7):7847-55

21. Wang L, Wei $Y$, Yan $Y$, Wang $H$, Yang J, Zheng Z, Zha J, Bo P, Tang $Y$, Guo X, Chen W, Zhu X, Ge L. CircDOCK1 suppresses cell apoptosis via inhibition of miR-196a-5p by targeting BIRC3 in OSCC. Oncol Rep 2018;39(3):951-66. doi: 10.3892/or.2017.6174

22. Li X, He J, Shao M, Cui B, Peng F, Li J, Ran Y, Jin D, Kong J, Chang J, Duan L, Yang X, Luo Y, Lu Y, Lin B, Liu T. Downregulation of miR218-5p promotes invasion of oral squamous cell carcinoma cells via activation of CD44-ROCK signaling. Biomed Pharmacother 2018;106:646-54. doi: 10.1016/j.biopha.2018.06.151

23. Kurihara-Shimomura M, Sasahira T, Shimomura H, Nakashima C, Kirita T. The Oncogenic Activity of miR-29b-1-5p Induces the Epithelial-Mesenchymal Transition in Oral Squamous Cell Carcinoma. J Clin Med 2019;8(2):273. doi: 10.3390/jcm8020273

24. Li J, Chen W, Luo L, Liao L, Deng X, Wang Y. The microRNA miR-29c$5 p$ inhibits cell proliferation and migration by targeting TMEM 98 in head and neck carcinoma. Aging (Albany NY) 2020;13(1):769-81. doi: 10.18632/aging.202183

25. Adeshakin FO, Adeshakin AO, Afolabi LO, Yan D, Zhang G, Wan $X$. Mechanisms for Modulating Anoikis Resistance in Cancer and the Relevance of Metabolic Reprogramming. Front Oncol 2021;11:626577. doi: 10.3389/fonc.2021.626577

26. Buchheit CL, Rayavarapu RR, Schafer ZT. The regulation of cancer cell death and metabolism by extracellular matrix attachment. Semin Cell Dev Biol 2012;23(4):402-11. doi: 10.1016/j.semcdb.2012.04.007

27. Dobashi Y, Watanabe Y, Miwa C, Suzuki S, Koyama S. Mammalian target of rapamycin: a central node of complex signaling cascades. Int J Clin Exp Pathol 2011;4(5):476-95.

28. Russell FM, Hardie DG. AMP-Activated Protein Kinase: Do We Need Activators or Inhibitors to Treat or Prevent Cancer? Int J Mol Sci 2020;22(1):186. doi: 10.3390/ijms22010186

29. Bhatti GK, Khullar N, Sidhu IS, Navik US, Reddy AP, Reddy PH, Bhatti JS. Emerging role of non-coding RNA in health and disease. Metab Brain Dis 2021;36(6):1119-134. doi: 10.1007/s11011-021-00739-y

30. Kim S, Lee JW, Park YS. The Application of Next-Generation Sequencing to Define Factors Related to Oral Cancer and Discover Novel Biomarkers. Life (Basel) 2020;10(10):228. doi: 10.3390/ life 10100228

31. Chen Z, Tao Q, Qiao B, Zhang L. Silencing of LINC01116 suppresses the development of oral squamous cell carcinoma by up-regulating microRNA-136 to inhibit FN1. Cancer Manag Res 2019;11:6043-59. doi: 10.2147/CMAR.S197583

32. He B, Lin X, Tian F, Yu W, Qiao B. MiR-133a-3p Inhibits Oral Squamous Cell Carcinoma (OSCC) Proliferation and Invasion by Suppressing COL1A1. J Cell Biochem 2018;119(1):338-46. doi: 10.1002/jcb.26182
33. Qin L, Zhan Z, Wei C, Li X, Zhang T, Li J. Hsa-circRNA-G004213 promotes cisplatin sensitivity by regulating miR-513b-5p/PRPF39 in liver cancer. Mol Med Rep 2021;23(6):421. doi: 10.3892/mmr.2021.12060

34. Lu C, Jia S, Zhao S, Shao X. MiR-342 regulates cell proliferation and apoptosis in hepatocellular carcinoma through Wnt/ $\beta$-catenin signaling pathway. Cancer Biomark 2019;25(1):115-26. doi:10.3233/ CBM-192399

35. Yang H, Li Q, Niu J, Li B, Jiang D, Wan Z, Yang Q, Jiang F, Wei P, Bai S. microRNA-342-5p and miR-608 inhibit colon cancer tumorigenesis by targeting NAA10. Oncotarget 2016;7(3):2709-20. doi: 10.18632/ oncotarget.6458

36. Wang C, Zhang W, Xing S, Wang Z, Wang J, Qu J. MiR-342-3p inhibits cell migration and invasion through suppressing forkhead box protein Q1 in ovarian carcinoma. Anticancer Drugs 2019;30(9):917-24. doi: 10.1097/CAD.0000000000000801

37. Monteiro LS, Diniz-Freitas M, Warnakulasuriya S, Garcia-Caballero T, Forteza-Vila J, Fraga M. Prognostic Significance of Cyclins A2, B1, D1, and E1 and CCND1 Numerical Aberrations in Oral Squamous Cell Carcinomas. Anal Cell Pathol (Amst) 2018;2018:7253510. doi: $10.1155 / 2018 / 7253510$

38. Khumukcham SS, Samanthapudi VSK, Penugurti V, Kumari A Kesavan PS, Velatooru LR, Kotla SR, Mazumder A, Manavathi B. Hematopoietic PBX-interacting protein is a substrate and an inhibitor of the APC/C-Cdc20 complex and regulates mitosis by stabilizing cyclin B1. J Biol Chem 2019;294(26):10236-252. doi: 10.1074/jbc. RA118.006733

39. Liu A, Zeng S, Lu X, Xiong Q, Xue Y, Tong L, Xu W, Sun Y, Zhang Z, Xu C. Overexpression of $\mathrm{G} 2$ and $\mathrm{S}$ phase-expressed-1 contributes to cell proliferation, migration, and invasion via regulating $\mathrm{p} 53 / \mathrm{FoxM} 1 /$ CCNB1 pathway and predicts poor prognosis in bladder cancer. Int J Biol Macromol 2019;123:322-34. doi: 10.1016/j.ijbiomac.2018.11.032

40. Wang Pu,Wei Libin,Ni Guangxiao.The effect of CCNB1 gene on the proliferation, invasion and migration of oral squamous cell carcinoma cells by regulating the PI3K/Akt signaling pathway. Journal of Practical Oncology 2020;34(02):144-49. doi: 10.11904 /j.issn.1002-3070.2020.02.011

41. Du R, Huang C, Liu K, Li X, Dong Z. Targeting AURKA in Cancer: molecular mechanisms and opportunities for Cancer therapy. Mol Cancer 2021;20(1):15. doi: 10.1186/s12943-020-01305-3

42. Dawei H, Honggang D, Qian W. AURKA contributes to the progression of oral squamous cell carcinoma (OSCC) through modulating epithelial-to-mesenchymal transition (EMT) and apoptosis via the regulation of ROS. Biochem Biophys Res Commun 2018;507(1-4):8390. doi: 10.1016/j.bbrc.2018.10.170

43. Yang Z, Liang X, Fu Y, Liu Y, Zheng L, Liu F, Li T, Yin X, Qiao X, Xu $X$. Identification of AUNIP as a candidate diagnostic and prognostic biomarker for oral squamous cell carcinoma. EBioMedicine 2019;47:44-57. doi: 10.1016/j.ebiom.2019.08.013

44. Feng CJ, Li HJ, Li JN, Lu YJ, Liao GQ. Expression of Mcm7 and Cdc6 in oral squamous cell carcinoma and precancerous lesions. Anticancer Res 2008;28(6A):3763-9. 